\title{
FOLIICOLOUS LICHENIZED FUNGI OF LOWLAND AMAZON FORESTS IN PANDO, BOLIVIA ${ }^{1}$
}

\author{
ADAM FLAKUS
}

\begin{abstract}
Foliicolous lichens are an important component of lowland tropical forests and are useful bioindicators of vegetation continuity or forest health. This study of these fungi in lowland Bolivian Amazon forests of Pando Department revealed the presence of 180 species, 101 of which are reported as new for Bolivia. The following 15 species are mentioned for the first time from South America: Anisomeridium guttuliferum Lücking, Byssolecania pluriseptata Breuss, Caprettia confusa Lücking \& Sipman, Chroodiscus submuralis Lücking, Coenogonium barbatum Lücking, Aptroot \& L. Umaña-Tenorio, Echinoplaca tetrapla (Zahlbr.) Lücking, Gyalectidium laciniatum Lücking, G. pallidum Herrera-Campos \& Lücking, Mazosia conica Sérus., Phylloblastia excavata P. M. McCarthy, Porina conica R. Sant., Porina subnucula Lücking \& Vězda, Porina aff. subpallescens Vězda, Strigula minuta Lücking and Trichothelium intermedium Lücking. The up-to-date list of foliicolous lichens of Bolivia now numbers 218 species, 52\% more than known previously. The foliicolous lichen biota of Pando, a substantial part of the Bolivian Amazon, has clear affinities to neighboring countries due to the significant proportion of species typical for lowland and submontane humid forests in the Neotropics. Analyses of the lichen diversity in the area suggest that its species composition is positively correlated with the presence of specific forest types.
\end{abstract}

Key words: Biodiversity, distribution, foliicolous lichens, Neotropics, South America

Adam Flakus, Laboratory of Lichenology, W. Szafer Institute of Botany, Polish Academy of Sciences, Lubicz 46, 31-512 Kraków, Poland; e-mail: a.flakus@botany.pl

\section{INTRODUCTION}

The Amazon Basin preserves the largest continuous area of humid tropical forests and is still considered one of the world's most species-rich hotspots, but unreasonable natural resource management is leading to irreversible damage of its ecosystems (e.g., Davidson et al. 2012). The Bolivian Amazon is in the northern and northeastern parts of the country within the departments of Beni, La Paz, Pando and Santa Cruz; its different forest communities form a mosaic of vegetation which offers a variety of potential habitats for foliicolous lichens (Navarro \& Maldonado 2002; Ibisch \& Mérida 2004; Navarro \& Ferreira 2007).

Pando Department is in the northernmost part of the Bolivian Amazon $\left(9^{\circ} 38^{\prime}-12^{\circ} 30^{\prime} \mathrm{S}\right.$, $\left.69^{\circ} 35^{\prime}-65^{\circ} 17^{\prime} \mathrm{W}\right)$, sharing borders with Brazil and Peru and covering $63,827 \mathrm{~km}^{2}$ at $90-289 \mathrm{~m}$

\footnotetext{
1 This paper is dedicated to Professor Tamás Pócs, on the occasion of his $80^{\text {th }}$ birthday.
}

a.s.1. (Fig. 1). Its mean annual temperature is $\mathrm{ca}$ $26^{\circ} \mathrm{C}$. Mean annual rainfall in the central part of the department is $1500-1850 \mathrm{~mm}$. In contrast, on the outskirts in the northeast (near Cobija) and northwest (near Nueva Esperanza) are very humid regions having 1900-3300 $\mathrm{mm}$ precipitation per year, with the maximum reaching 3600-5000 mm (Anonymous 1997; Navarro \& Maldonado 2002; Ibisch \& Mérida 2004; Navarro 2011; http://www. ine.gob.bo). The department has only one area protected at the national level: Reserva Nacional de Vida Silvestre Amazónica Manuripi in the southwest of the department.

Pando boasts a diverse system of vegetation including several types of riverine and terrestrial forests (Navarro \& Maldonado 2002; Navarro 2003; Mostacedo et al. 2006), and is considered one of the most important biodiversity centers in Bolivia (Montambault 2002; Ibisch \& Mérida 2004). In particular, its northeastern part in the 


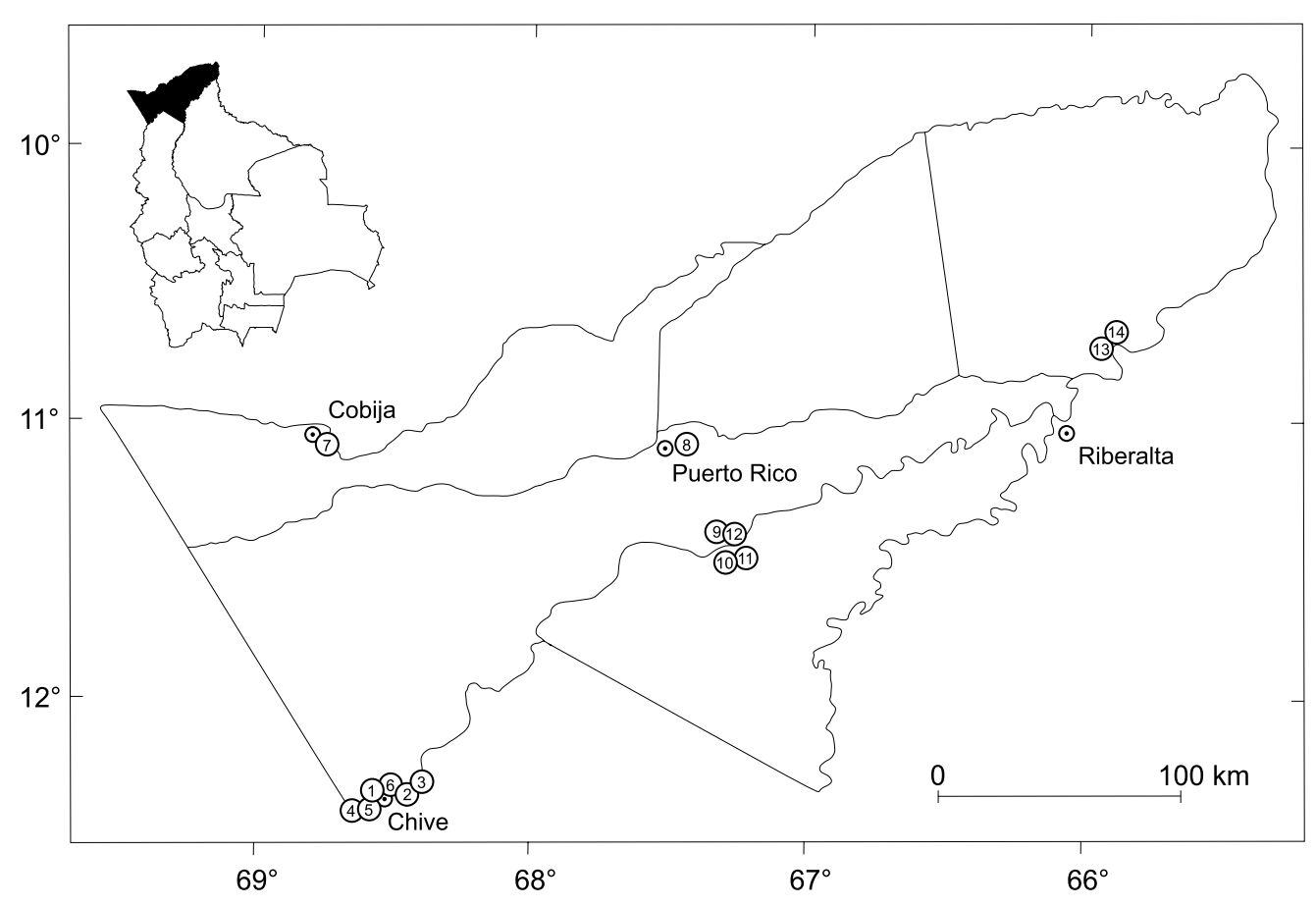

Fig. 1. Map of Pando showing lichen sampling locations.

Precambrian Shield region, which is covered by Tapajoz and Madeira vegetation, is ranked as the biologically richest and least disturbed forest areas in the whole Bolivian Amazon and has been strongly recommended for protection as a strict reserve (Montambault 2002; Ibisch \& Mérida 2004; Navarro 2011).

Due to its geographical location and significant share of natural forest, Pando is an essential part of the Bolivian Amazon, but its lichen biota has received almost no attention so far (Flakus \& Farkas 2013). The importance of this area is reinforced by a recent survey in an adjacent area of the Peruvian Amazon, which documented the second-highest number of Graphidaceae ever reported for a single site anywhere (Rivas Plata \& Lücking 2013).

This paper on foliicolous lichens occurring in that area is intended to help fill a large information gap and improve our knowledge of the biodiversity and distribution of lichens in Bolivia. It provides many new national records, including species mentioned here for the first time from South America.
Several undescribed species were found in the examined material. They need further attention and will be treated in forthcoming papers.

\section{MATERIAL AND METHODS}

The data are based on specimens I collected in Bolivia during investigations there in 2006. At each studied locality I sampled the foliicolous lichens of an area of homogeneous forest vegetation measuring 50-100 $\mathrm{m}^{2}$. The forest types at each locality were categorized according to the vegetation map of Bolivia by Navarro and Ferreira (2007).

The material was identified by standard methods. Morphology was observed with a Nikon SMZ800 stereomicroscope. Microscopy preparations were studied in water, a solution of potassium hydroxide, and for ascus structure in Lugol's iodine solution, using a Nikon Eclipse 80i (DIC) compound microscope. Thin-layer chromatography analyses were done according to Orange et al. (2001). Voucher specimens are deposited in KRAM (Kraków), LPB (La Paz) and my private collection.

To compare foliicolous lichen composition between sites, Jaccard's similarity index between each pair of 
sites (Jaccard 1908) and the number of shared species were calculated, followed by non-metric multidimensional scaling ordination (NMDS) standardized for binary data using Jaccard's dissimilarity index as the distance measure, and subsequent auto-transformation (Minchin 1987). Additionally, hierarchical cluster analysis for binary data was run using the average linkage agglomeration method (Crawley 2013). Sites 3 and 5 were excluded from the analyses because of their low diversity. All statistical calculations were run with environment R ver. 3.0.2 (http://www.R-project.org) using the stats and vegan packages. To evaluate possible undersampling, the Chao 2 index estimating the total species richness in the area was calculated (Chao et al. 2009).

Localities are indicated in the species list and on the map (Fig. 1) by numbers corresponding to the list below, with forest symbols according to Navarro and Ferreira (2007) in parentheses. All specimens examined are from Pando Department, Bolivia.

\section{LIST OF LOCALITIES}

1. Prov. Manuripi, Reserva Nacional de Vida Silvestre Amazónica Manuripi, Chive, 12 $23^{\prime} 47^{\prime \prime} \mathrm{S}$, $68^{\circ} 35^{\prime} 28^{\prime \prime} \mathrm{W}, 179 \mathrm{~m}, 18$ May 2006, anthropogenically disturbed terrestrial Amazon forest of northeast Pando (a20/a1aii);

2. Prov. MANURIPI, Reserva Nacional de Vida Silvestre Amazónica Manuripi, Bajada near Chive by río Madre de Dios, $12^{\circ} 24^{\prime} 03^{\prime \prime} \mathrm{S}, 68^{\circ} 26^{\prime} 45^{\prime \prime} \mathrm{W}, 170 \mathrm{~m}$, 19 May 2006, white-water-flooded Amazon forest (Várzea) (a29);

3. Prov. Manuripi, Reserva Nacional de Vida Silvestre Amazónica Manuripi, Metale near Chive by río Madre de Dios, $12^{\circ} 22^{\prime} 33^{\prime \prime} \mathrm{S}, 68^{\circ} 25^{\prime} 21^{\prime \prime} \mathrm{W}, 170 \mathrm{~m}$, 19 May 2006, white-water-flooded Amazon forest (Várzea) (a29);

4. Prov. ManuripI, Reserva Nacional de Vida Silvestre Amazónica Manuripi, near Puerto Heath, $12^{\circ} 29^{\prime} 38^{\prime \prime} \mathrm{S}, 68^{\circ} 37^{\prime} 28^{\prime \prime} \mathrm{W}, 170 \mathrm{~m}, 20$ May 2006, whitewater-flooded Amazon forest (Várzea) (a29);

5. Prov. Manuripi, Reserva Nacional de Vida Silvestre Amazónica Manuripi, near Nueva España, $12^{\circ} 28^{\prime} 32^{\prime \prime} \mathrm{S}, 68^{\circ} 35^{\prime} 33^{\prime \prime} \mathrm{W}, 170 \mathrm{~m}, 20$ May 2006, whitewater-flooded Amazon forest (Várzea) (a29);

6. Prov. MAnuripi, Reserva Nacional de Vida Silvestre Amazónica Manuripi, Chive, 12²3'19"S, 68 34'39"W, 157 m, 21 May 2006, anthropogenically disturbed terrestrial Amazon forest of northeast Pando (a20/a1aii);
7. Prov. Nicolas Suarez, Parque Ecologico Cobija, near Cobija, $11^{\circ} 02^{\prime} 16^{\prime \prime} \mathrm{S}, 68^{\circ} 45^{\prime} 31^{\prime \prime} \mathrm{W}, 191 \mathrm{~m}$, 23 May 2006, anthropogenically disturbed white-waterflooded Pando Amazon forest (a20/a10);

8. Prov. MAnuriPI, Reserva Nacional de Vida Silvestre Amazónica Manuripi, Puerto Rico, $11^{\circ} 06^{\prime} 12^{\prime \prime} \mathrm{S}$, $67^{\circ} 31^{\prime} 14^{\prime \prime} \mathrm{W}, 191 \mathrm{~m}, 25$ May 2006, terrestrial Amazon forest of northeast Pando (a1aii);

9. Prov. MANURIPI, Reserva Nacional de Vida Silvestre Amazónica Manuripi, Puerto Madre de Dios, $11^{\circ} 27^{\prime} 38^{\prime \prime} \mathrm{S}, 67^{\circ} 15^{\prime} 56^{\prime \prime} \mathrm{W}, 166 \mathrm{~m}, 26$ May 2006 , whitewater-flooded Amazon forest (Várzea) (a29);

10. PRov. MAdRE DE Dios, Reserva Nacional de Vida Silvestre Amazónica Manuripi, near Puerto Madre de Dios by río Madre de Manupare, $11^{\circ} 31^{\prime} 37^{\prime \prime} \mathrm{S}$, $67^{\circ} 17^{\prime} 29^{\prime \prime} \mathrm{W}, 155$ m, 27 May 2006, white-water-flooded Amazon forest (Várzea) (a29);

11. Prov. MAdRE DE Dios, Reserva Nacional de Vida Silvestre Amazónica Manuripi, near Puerto Madre de Dios by río Madre de Manupare, $11^{\circ} 30^{\prime} 56^{\prime \prime} \mathrm{S}$, $67^{\circ} 16^{\prime} 07^{\prime \prime} \mathrm{W}, 153 \mathrm{~m}, 27$ May 2006, white-water-flooded Amazon forest (Várzea) (a29);

12. Prov. MANURIPI, Reserva Nacional de Vida Silvestre Amazónica Manuripi, Puerto Madre de Dios, $11^{\circ} 27^{\prime} 38^{\prime \prime} \mathrm{S}, 67^{\circ} 15^{\prime} 56^{\prime \prime} \mathrm{W}, 166 \mathrm{~m}, 27$ May 2006, whitewater-flooded Amazon forest (Várzea) (a29);

13. Prov. Federico RomAn, near Santa Cruzito, $10^{\circ} 43^{\prime} 03^{\prime \prime} \mathrm{S}, 65^{\circ} 55^{\prime} 05^{\prime \prime} \mathrm{W}, 149 \mathrm{~m}, 1$ June 2006 , blackwater-flooded Amazon forest (Igapó) of east Pando (a6bii);

14. Prov. Federico Roman, near Santa Cruzito, $10^{\circ} 41^{\prime} 44^{\prime \prime} \mathrm{S}, 65^{\circ} 54^{\prime} 54^{\prime \prime} \mathrm{W}, 151 \mathrm{~m}, 1$ June 2006 , blackwater-flooded Amazon forest (Igapó) of east Pando (a6bii).

\section{RESULTS AND DISCUSSION}

\section{BIOGEOGRAPHIC RESULTS}

The first records of foliicolous lichens in Bolivia were given by Santesson (1952). More data were reported by Ferraro (2002), Lücking (2008), Flakus and Lücking (2008), Farkas (2010) and Flakus and Farkas (2013). Before this study, 117 foliicolous species were reported from the country. Only six species of foliicolous lichens were reported previously from Pando Department (Flakus \& Farkas 2013), an important part of the Bolivian Amazon.

Based on recent collections of 180 species discovered in Pando (Table 1), including 101 records 
new for the country, the latest list of foliicolous lichens of Bolivia includes 218 species, increasing the number of taxa by $52 \%$. In view of Bolivia's high ecosystem diversity (Josse et al. 2003) and the large number of collections from various localities now being prepared (Flakus unpubl.), our current knowledge of its foliicolous lichen biota on the national scale must be far from complete.

Here I report fifteen species for the first time from South America. Previously they have been placed in three main biogeographic groups: (1) Neotropical species having a Central American distribution pattern, (2) Pantropical species known outside of continental South America, and (3) species known from few localities in the Paleotropics and/or Australia. The first group contains nine species recently described from extensively studied areas in Central America: Anisomeridium guttuliferum (previously known from Costa Rica), Byssolecania pluriseptata (Costa Rica), Caprettia confusa (Costa Rica), Chroodiscus submuralis (Costa Rica), Coenogonium barbatum (Costa Rica), Gyalectidium laciniatum (Costa Rica), G. pallidum (Mexico), Strigula minuta (Costa Rica) and Trichothelium intermedium (Mexico) (Santesson 1952; Ferraro et al. 2001; HerreraCampos \& Lücking 2002; Herrera-Campos et al. 2004; Rivas Plata et al. 2006; Breuss \& Neuwirth 2007; Lücking 2008). The second group is represented by species known from scattered localities across the tropics in Central America, Australia and the Paleotropics, and includes Echinoplaca tetrapla (Costa Rica, Mexico, Thailand), Mazosia conica (Costa Rica, Mexico, Papua New Guinea) and Porina conica (Africa, Australasia, Asia) (Santesson 1952; Sipman 1993; Aptroot et al. 1997; Boonpragob et al. 1998; Lücking \& Vězda 1998; Papong et al. 2007; Lücking 2008). The third group includes Phylloblastia excavata recently described from Australia (McCarthy 2010) and Porina subnucula known from two collections in tropical Africa and Australia (Lücking \& Vězda 1998), as well as $P$. aff. subpallescens reported only from the locus classicus in Tanzania (Vězda 1975). The Bolivian specimen of $P$. subpallescens fits Vězda's original description but its photobiont had different, irregularly arranged angular-rounded cells, so it may represent another undescribed species. The specimen was very small and did not lend itself to description; I shall not to describe it until more collections are discovered.

\section{ECOLOGICAL OBSERVATIONS}

Foliicolous lichen diversity (Fig. 2) ranged from 10 to 72 species per studied site and was highest in undisturbed Igapó forest (site 13) in the Precambrian Shield area of eastern Pando (Fig. 3). The common foliicolous lichens, found in at least half localities, numbered only 18 species $(10 \%$ of the total): Anisomeridium foliicola, Arthonia accolens, Bacidina neotropica, Byssolecania hymenocarpa, Chroodiscus coccineus, Flavobathelium epiphyllum, Gyalectidium filicinum, G. pallidum, Lyromma ornatum, Mazosia dispersa, M. rotula, Phyllobathelium leguminosae, Porina alba, P. papillifera, Sporopodium leprieurii, Strigula maculata, S. phyllogena and Trichothelium pallescens. In contrast, very rare species, found only in one or two localities, formed $53 \%$ of the local lichen biota and were represented by 96 species (Table 1).

The species composition in Pando is characteristic for Neotropical lowland humid Amazon forests (e.g., Lücking 1995, 1997, 1998, 2008; Lücking \& Kalb 2000). The foliicolous lichen biota consists of 79 species (44\%) previously classified by Lücking $(1997,2000)$ as indicators of typical lowland or lowland to submontane forest. Thirty of those (17\%) represent the lowland element: Bacidina pseudohyphophorifera, Bapalmuia nigrescens, B. palmularis, Byssolecania fumosonigricans, B. hymenocarpa, B. variabilis, B. amazonicum, Coenogonium dilucidum, $C$. siquirrense, C. subluteum, Cryptothecia filicina, Eugeniella psychotriae, Fellhanera muhlei, F. verrucifera, Lyromma palmae, Malmidea trailiana, Mazosia praemorsa, M. pseudobambusae, M. rubropunctata, M. tenuissima, M. tumidula, Phyllobathelium taxteri, Phyllogyalidea epiphylla, Porina atriceps, P. fusca, P. radiata, Sporopodium antonianum, Strigula janeirensis, Trichothelium pallescens and T. sipmanii. The most common of the 49 species characteristic of lowland to submontane forest are 

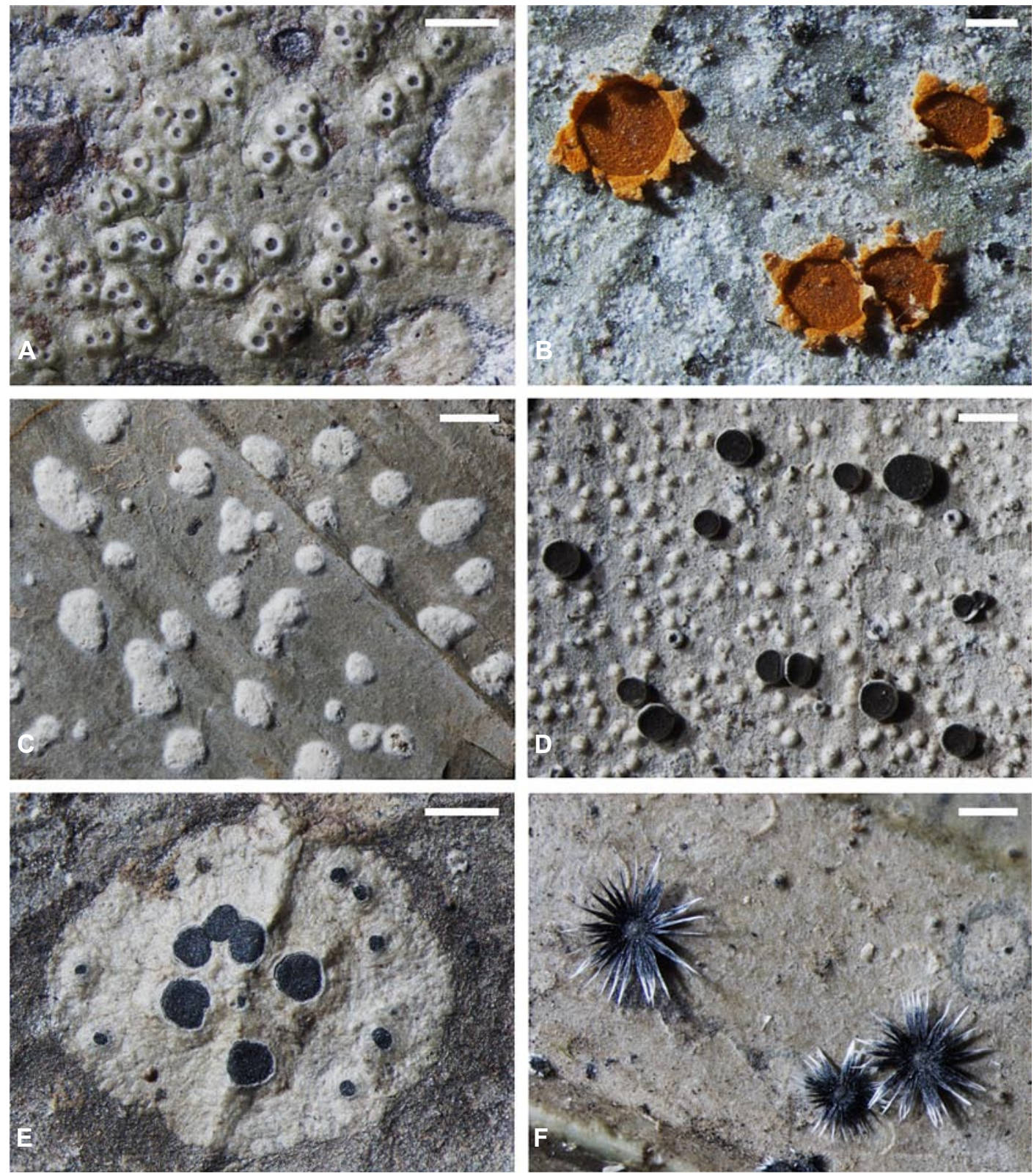

Fig. 2. Morphological diversity of foliicolous lichens in Pando. A - Caleniopsis aggregata (R. Sant.) Lücking, Sérus. \& Vězda, B - Chroodiscus coccineus (Leight.) Müll. Arg., C - Cryptothecia filicina (Ellis \& Everh.) Lücking, Thor, Aptroot \& Kalb, D Eugeniella psychotriae (Müll. Arg.) Lücking, Sérus. \& Kalb, E - Mazosia conica Sérus., F - Trichothelium argenteum Lücking $\&$ L. I. Ferraro. Scale bars: A, D, E, F $=500 \mu \mathrm{m} ; \mathrm{B}=250 \mu \mathrm{m} ; \mathrm{C}=1000 \mu \mathrm{m}$.

Aderkomyces heterellus, Anisomeridium foliicola, Arthonia accolens, Aulaxina minuta, Bacidina neotropica, Byssoloma minutissimum, B. tricholomum, Coenogonium hypophyllum, Fellhanera fuscatula,
Mazosia dispersa, M. melanophtalma, M. paupercula, M. pilosa, M. rotula, Phyllobathelium leguminosae, Flavobathelium epiphyllum, Porina atrocoerulea, P. epiphylla, P. karnatakensis, 
Table 1. Foliicolous lichen taxa identified in Pando: ${ }^{*}-$ new for Bolivia, ${ }^{* *}$ - new for South America.

\begin{tabular}{|c|c|c|}
\hline Species name & Locality number & Collection number \\
\hline $\begin{array}{l}\text { Aderkomyces heterellus (Stirt.) Lücking, Sérus. } \\
\text { \& Vězda }\end{array}$ & $10,13,14$ & $6728,7289,7378$ \\
\hline Anisomeridium foliicola R. Sant. \& Tibell & $\begin{array}{l}1,2,4,6,10,11 \\
12,13\end{array}$ & $\begin{array}{l}5905,5909,5921.2,5994,6001,6016.1,6050, \\
6065,6075,6268,6272,6289,6415,6488.1, \\
6724.1,6748,6757,6767.1,6776.2,6781,6867, \\
7082,7199\end{array}$ \\
\hline Anisomeridium guttuliferum Lücking & 2 & $5978.1,5982,6023.1,6035.1$ \\
\hline "Anisomeridium prolongatum Lücking & 2 & 5978.2 \\
\hline Arthonia accolens Stirt. & $\begin{array}{l}1,3,5,6,10 \\
13,14\end{array}$ & $\begin{array}{l}5886,6180,6186,6201,6353,6395,6416,6427, \\
6452,6487,6492,6713,6741,6759,7222,7304, \\
7318,7400,7403,7458\end{array}$ \\
\hline "Arthonia aciniformis Stirt. & $6,9,13,14$ & $6412,6434.3,6470,6625.1,7138,7147,7348.1$ \\
\hline Arthonia cyanea Müll. Arg. var. cyanea f. cyanea & 5,6 & $6324,6328,6361,6384,6453,6500$ \\
\hline Arthonia leptosperma (Müll. Arg.) R. Sant. & $2,11,13$ & $\begin{array}{l}6007,6059,6794,6797.1,6798.1,6862,6863, \\
6868,6888,6914,6923,7109\end{array}$ \\
\hline "Arthonia orbygniae (H. B. P. Upadhyay) Matzer & 14 & $7426,7438.1$ \\
\hline "Arthonia palmulacea (Müll. Arg.) R. Sant. & 14 & 7452.1 \\
\hline Aspidothelium fugiens (Müll. Arg.) R. Sant. & 14 & 7348 \\
\hline Asterothyrium monosporum Müll. Arg. & 9 & 6650.3 \\
\hline $\begin{array}{l}\text { Asterothyrium rondoniense Bat. \& H. Maia ex } \\
\text { Henssen \& Lücking }\end{array}$ & 9,12 & 6612,6980 \\
\hline Aulaxina microphana (Vain.) R. Sant. & 9,12 & $5939,6078.2,7049$ \\
\hline "Aulaxina minuta $\mathrm{R}$. Sant. & $12,13,14$ & $\begin{array}{l}6987,7044,7186.1,7419.1,7328,7337,7347.2, \\
7462\end{array}$ \\
\hline "Aulaxina submuralis Kalb \& Vězda & 2 & 5947.1 \\
\hline Bacidina apiahica (Müll. Arg.) Vězda & $1,2,7,13$ & $5860.2,6041,6549,7282$ \\
\hline "Bacidina hypophylla Lücking \& Kalb & 10,14 & $6710.1,7343.1,7460$ \\
\hline Bacidina neotropica Lücking & $\begin{array}{l}1,2,4,7,9,11 \\
12,13\end{array}$ & $\begin{array}{l}5867,5950,6236,6255,6267,6294.3,6501, \\
6610.2,6632,6643,6673,6698,6828,6841.1, \\
6844.1,6876,6878,6923.1,6992.1,7162,7189, \\
7232\end{array}$ \\
\hline Bacidina pallidocarnea (Müll. Arg.) Vězda & 7,8 & $6470.2,6556$ \\
\hline $\begin{array}{l}\text { *Bacidina pseudohyphophorifera (Lücking } \\
\text { \& Sérus.) Lücking }\end{array}$ & $6,13,14$ & $6420,6428,7267,7443$ \\
\hline *Bapalmuia costaricensis Lücking \& Kalb & $1,2,7,11$ & $5922,6045,6490,6947$ \\
\hline "Bapalmuia lineata Lücking \& Kalb & $2,4,7,11,13$ & $\begin{array}{l}5940,5968,5996.1,6005,6034,6047,6051, \\
6056.1,6068,6302,6536.1,6856,6887,6954, \\
7203\end{array}$ \\
\hline $\begin{array}{l}\text { “Bapalmuia nigrescens (Müll. Arg.) M. Cáceres } \\
\text { \& Lücking }\end{array}$ & 13 & 7238.1 \\
\hline "Bapalmuia palmularis (Müll. Arg.) Sérus. & $11,13,14$ & $6963,7117,7122,7159,7261,7345,7404.2$ \\
\hline $\begin{array}{l}\text { Byssolecania fumosonigricans (Müll. Arg.) R. } \\
\text { Sant. }\end{array}$ & $1,11,12,13$ & $\begin{array}{l}5911,5926.1,6788,6799,6841,6854,6889, \\
6925,6948,7079,7151\end{array}$ \\
\hline $\begin{array}{l}\text { Byssolecania hymenocarpa (Vain.) Kalb, Vězda } \\
\text { \& Lücking }\end{array}$ & $\begin{array}{l}1,2,3,4,6,7,9 \\
10,12,14\end{array}$ & $\begin{array}{l}5852,5852.1,5861.1,5880,5953,5980,6000, \\
6017,6023,6025,6032,6040,6058,6066,6073, \\
6074,6078,6080,6199,6251,6282.1,6286, \\
6306,6434.2,6490.3,6438,6446,6526,6528, \\
6552.3,6643.1,6708.1,6731,6676.1,7033, \\
7039,7072.1,7099,7457.1\end{array}$ \\
\hline
\end{tabular}


Table 1. Continued.

\begin{tabular}{|c|c|c|}
\hline Species name & Locality number & Collection number \\
\hline${ }^{* *}$ Byssolecania pluriseptata Breuss & 11,13 & $6945,7159.1,7190,7225$ \\
\hline Byssolecania variabilis Vězda, Kalb \& Lücking & 2,11 & $5948,6019,6799.1$ \\
\hline "Byssoloma absconditum Farkas \& Vězda & 13 & 7281 \\
\hline "Byssoloma amazonicum Kalb \& Vězda & 14 & 7410,7439 \\
\hline Byssoloma chlorinum (Vain.) Zahlbr. & $3,6,8,10,13,14$ & $\begin{array}{l}6170,6179,6190,6203,6377,6386,6399 \\
6405.3,6410,6415.1,6425,6426,6485,6546.1 \\
6587.1,6749,7186,7216,7334,7371,7424\end{array}$ \\
\hline $\begin{array}{l}\text { "Byssoloma citricola (Maubl.) Lücking, Sérus. } \\
\text { \& R. Sant. }\end{array}$ & $1,3,6,8$ & $5877,6175,6481,6588.2$ \\
\hline "Byssoloma humboldtianum Lücking \& Kalb & 14 & $7340.2,7354.1,7420$ \\
\hline Byssoloma leucoblepharum (Nyl.) Vain. & 6,14 & $6382,6418.1,6426.2,6429,6460.1,7457$ \\
\hline "Byssoloma minutissimum Kalb \& Vězda & $1,7,9,11,13,14$ & $\begin{array}{l}5887.1,6457.2,6513.1,6623,6839.1,7124 \\
7150,7177,7240,7250,7262,7293,7350.1\end{array}$ \\
\hline "Byssoloma tricholomum (Mont.) Zahlbr. & $5,6,13,14$ & $\begin{array}{l}6336,6343,6354,6358,6429.2,7240,7274, \\
7359,7411,7448\end{array}$ \\
\hline Calenia phyllogena (Müll. Arg.) R. Sant. & 2 & 6054 \\
\hline${ }^{*}$ Calenia thelotremella Vain. & 1,2 & $5921,6030.1$ \\
\hline${ }^{*}$ Calenia triseptata Zahlbr. & 2 & $5931,5946,5955,5971,6009,6027$ \\
\hline $\begin{array}{l}\text { "Caleniopsis aggregata (R. Sant.) Lücking, Sérus. } \\
\text { \& Vězda }\end{array}$ & 2,7 & $5941,5960,5988,6037,6067,6483$ \\
\hline $\begin{array}{l}\text { "Caleniopsis conspersa (Stirt.) Lücking, Sérus. } \\
\text { \& Vězda }\end{array}$ & 14 & $7409.1,7351$ \\
\hline${ }^{*}$ Caleniopsis laevigata (Müll. Arg.) Vězda \& Poelt & 13,14 & $7220.1,7346,7396.1,7419$ \\
\hline Calopadia foliicola (Fée) Vězda & $1,6,8$ & $5874.1,6406.1,6421,6567,6586,6597$ \\
\hline Calopadia fusca (Müll. Arg.) Vězda & 5 & 6359 \\
\hline Calopadia perpallida (Nyl.) Vězda & 6 & $6406,6482,6496$ \\
\hline Calopadia puiggarii (Müll. Arg.) Vězda & 5 & 6329 \\
\hline${ }^{* * *}$ Caprettia confusa Lücking \& Sipman & $2,4,9,11,12$ & $6003,6300,6303,6616,6633,6690,6921,6967$ \\
\hline "Chroodiscus australiensis Vězda \& Lumbsch & 14 & 7382,7466 \\
\hline Chroodiscus coccineus (Leight.) Müll. Arg. & $\begin{array}{l}1,2,4,5,6,7,9 \\
12,13,14\end{array}$ & $\begin{array}{l}5885,5931.2,5937,5949,6026.2,6079.1,6292, \\
6327,6330,6339,6340,6341,6351,6356,6357, \\
6364,6424,6430,6436,6437,6463.1,6484, \\
6397,6520,6679,6684,6983,7036,7047,7156, \\
7183,7291,7329,7349,7351,7355,7382.1, \\
7392,7399,7412,7422,7425,7441\end{array}$ \\
\hline${ }^{* *}$ Chroodiscus submuralis Lücking & $10,13,14$ & $6758,7170,7398.1$ \\
\hline $\begin{array}{l}{ }^{* *} \text { Coenogonium barbatum Lücking, Aptroot } \\
\text { \& L. Umaña-Tenorio }\end{array}$ & 6 & 6422.1 \\
\hline${ }^{*}$ Coenogonium ciliatum Kalb \& Lücking & 13 & $7103,7258.1,7283$ \\
\hline $\begin{array}{l}\text { "Coenogonium dilucidum (Kremp.) Kalb } \\
\text { \& Lücking }\end{array}$ & 9 & 6685 \\
\hline $\begin{array}{l}\text { "Coenogonium fallaciosum (Müll. Arg.) Kalb } \\
\text { \& Lücking }\end{array}$ & 13 & 7105 \\
\hline "Coenogonium flavoviride M. Cáceres \& Lücking & 6 & 6489 \\
\hline "Coenogonium geralense (Henn.) Lücking & $6,10,11,13$ & $\begin{array}{l}6375,6405,6405.1,6428.1,6457.1,6472,6729, \\
6804,7188\end{array}$ \\
\hline $\begin{array}{l}\text { "Coenogonium hypophyllum (Vězda) Kalb } \\
\text { \& Lücking }\end{array}$ & $2,4,10,13,14$ & $5956,6275,6721,7200.1,7336,7460.1$ \\
\hline${ }^{*}$ Coenogonium interpositum $\mathrm{Nyl}$ & 14 & $7332,7439.1$ \\
\hline
\end{tabular}


Table 1. Continued.

\begin{tabular}{|c|c|c|}
\hline Species name & Locality number & Collection number \\
\hline${ }^{*}$ Coenogonium leprieurii (Mont.) Nyl. & $2,10,11,13,14$ & $\begin{array}{l}6034.2,6746,6798,6933,7104,7126,7197, \\
7258,7277,7284,7468\end{array}$ \\
\hline "Coenogonium minimum (Müll. Arg.) Lücking & 11 & 6816 \\
\hline "Coenogonium siquirrense (Lücking) Lücking & 6,13 & $6405.1,7145$ \\
\hline Coenogonium subluteum (Rehm) Kalb \& Lücking & $1,6,7,8,11,12$ & $\begin{array}{l}5876.1,5912,6392,6519,6575,6576,6587, \\
6595,6598,6600,6869,7067\end{array}$ \\
\hline $\begin{array}{l}\text { "Cryptothecia filicina (Ellis \& Everh.) Lücking, } \\
\text { Thor, Aptroot \& Kalb }\end{array}$ & 13,14 & $\begin{array}{l}\text { 7202, 7202.1, 7265, 7271, 7347, 7361, 7367, } \\
7374,7393,7395,7405,7395.1\end{array}$ \\
\hline${ }^{*}$ Echinoplaca campanulata Kalb \& Vězda & 8,9 & $6581,6642.1,6659.1$ \\
\hline Echinoplaca epiphylla Fée & 3,9 & $6193,6682.2$ \\
\hline Echinoplaca leucotrichoides (Vain.) R. Sant. & 2,13 & 5985,7226 \\
\hline Echinoplaca pellicula (Müll. Arg.) R. Sant. & $1,2,3,6,8$ & $\begin{array}{l}5920.2,6033,6074.2,6181,6184,6416,6426, \\
6468,6577,6581.1\end{array}$ \\
\hline${ }^{* *}$ Echinoplaca tetrapla (Zahlbr.) Lücking & 2 & 5979.2 \\
\hline $\begin{array}{l}\text { "Eugeniella corallifera (Lücking) Lücking, Sérus. } \\
\text { \& Kalb }\end{array}$ & 13 & 7187.1 \\
\hline $\begin{array}{l}\text { *Eugeniella leucocheila (Tuck.) Lücking, Sérus. } \\
\text { \& Kalb }\end{array}$ & 13,14 & $7171,7206,7206.1,7290,7324,7423$ \\
\hline $\begin{array}{l}\text { "Eugeniella psychotriae (Müll. Arg.) Lücking, } \\
\text { Sérus. \& Kalb }\end{array}$ & 13,14 & $7207,7218,7219,7421.1,7430,7446$ \\
\hline "Fellhanera bouteillei (Desm.) Vězda & 9,12 & 6649,6972 \\
\hline Fellhanera fuscatula (Müll. Arg.) Vězda & $7,9,11,13$ & $\begin{array}{l}6474.1,6482.1,6674,6801.2,6920,6930, \\
6948.1,7136,7140,7308\end{array}$ \\
\hline "Fellhanera longispora Lücking & 7,9 & $6524,6525.1,6530.1,6630,6650$ \\
\hline${ }^{*}$ Fellhanera muhlei Lücking & 6 & 6459.1 \\
\hline${ }^{*}$ Fellhanera punctata Lücking & 13,14 & 7307,7406 \\
\hline${ }^{*}$ Fellhanera semecarpi (Vain.) Vězda & 9 & 6683.5 \\
\hline Fellhanera stanhopeae (Müll. Arg.) Lücking & 6,9 & $6459,6636,6651.4,6675.1$ \\
\hline${ }^{*}$ Fellhanera verrucifera Lücking & 13,14 & 7251,7350 \\
\hline $\begin{array}{l}\text { "Flavobathelium epiphyllum Lücking, Aptroot } \\
\text { \& Thor }\end{array}$ & $\begin{array}{l}1,6,9,10,11 \\
12,13\end{array}$ & $\begin{array}{l}5904,5905.1,5908.1,5919.1,6401,6414.1, \\
6488,6619,6624.2,6626.1,6670,6695,6709, \\
6757.2,6760,6761,6766.1,6775,6869.2, \\
7070.1,7237,7319\end{array}$ \\
\hline $\begin{array}{l}\text { Gyalectidium catenulatum (Cavalc. \& A. A. Silva) } \\
\text { L. I. Ferraro, Lücking \& Sérus. }\end{array}$ & 12 & 6979 \\
\hline Gyalectidium filicinum Müll. Arg. & $\begin{array}{l}1,2,4,7,9,10 \\
12,13\end{array}$ & $\begin{array}{l}5861.2,5903,5913,5917,5979.1,6027.1,6086, \\
6237,6281.1,6295,6489.1,6514,6671.2,6712, \\
6974,7124.1,7249,7272\end{array}$ \\
\hline "Gyalectidium imperfectum Vězda & 6 & 6409,6483 \\
\hline${ }^{* * G}$ Galectidium laciniatum Lücking & 11,12 & $6836,7008,7040$ \\
\hline $\begin{array}{l}\text { **alectidium pallidum Herrera-Campos } \\
\text { \& Lücking }\end{array}$ & $\begin{array}{l}1,2,4,5,11,12 \\
13,14\end{array}$ & $\begin{array}{l}5870,5891.3,5897,5900.1,6022,6237.1, \\
6295.1,6308,6322,6323,6801.1,6805,6851, \\
6968,6977,7018,7059,7089,7201,7210,7252, \\
7303,7459\end{array}$ \\
\hline "Lasioloma arachnoideum (Kremp.) R. Sant. & 6 & 6495 \\
\hline Lyromma coronatum Flakus \& Farkas & 1,2 & 5925.1, 6018 (Flakus \& Farkas 2013) \\
\hline Lyromma dolicobelum Cavalc. & 8,13 & $\begin{array}{l}\text { 6570, 7107, 7111, 7123, 7127, 7157, 7160, 7181, } \\
\text { 7191, } 7257 \text { (Flakus \& Farkas 2013) }\end{array}$ \\
\hline Lyromma nectandrae Bat. \& H. Maia & $6,11,13$ & $\begin{array}{l}\text { 6457, 6802, 7161, 7193.1, 7215, 7260, } 7298 \\
\text { (Flakus \& Farkas 2013) }\end{array}$ \\
\hline
\end{tabular}


Table 1. Continued.

\begin{tabular}{|c|c|c|}
\hline Species name & Locality number & Collection number \\
\hline Lyromma ornatum Lücking, Kalb \& Sérus. & $\begin{array}{l}1,2,3,6,8,11 \\
13,14\end{array}$ & $\begin{array}{l}5882,5984,6019.1,6176,6407,6407.1,6455 \\
6460,6554,6565,6865,7193,7314,7349.1 \\
\text { (Flakus \& Farkas 2013) }\end{array}$ \\
\hline $\begin{array}{l}\text { Lyromma palmae (Calvac. \& A. A. Silva) Lücking } \\
\text { \& Sérus. }\end{array}$ & $2,9,12,13$ & $\begin{array}{l}\text { 5989.3, 6662, 6976, 7046, } 7314.2 \text { (Flakus \& Far- } \\
\text { kas 2013) }\end{array}$ \\
\hline Lyromma pilosum Lücking & $1,2,7,13$ & $\begin{array}{l}\text { 5903.2, 5975, 6019.2, 6440, } 6719 \text { (Flakus \& Far- } \\
\text { kas 2013) }\end{array}$ \\
\hline $\begin{array}{l}\text { "Malmidea trailiana (Müll. Arg.) Kalb, Rivas Plata } \\
\text { \& Lumbsch }\end{array}$ & 13 & 7153 \\
\hline${ }^{* * *}$ Mazosia conica Sérus. & 1,2 & $5861,5866,5873,5902,5977.1$ \\
\hline Mazosia dispersa (J. Hedrick) R. Sant. & $1,2,5,6,7,10,14$ & $\begin{array}{l}5872,5936,6039,6363,6438,6530,6779.1, \\
7464.1\end{array}$ \\
\hline Mazosia melanophtalma (Müll. Arg.) R. Sant. & $1,3,4,6,10,13$ & $\begin{array}{l}5877.1,6196,6243,6250,6293,6486.1,6720.3, \\
7194\end{array}$ \\
\hline Mazosia paupercula (Müll. Arg.) R. Sant. & $2,4,6$ & $6013.2,6282,6296,6434,6445$ \\
\hline Mazosia phyllosema (Nyl.) Zahlbr. & $1,8,12,13$ & $5889,6604,6998.1,7025,7317$ \\
\hline "Mazosia pilosa Kalb \& Vězda & $6,13,14$ & $6417,6486.2,6389,7149,7333$ \\
\hline 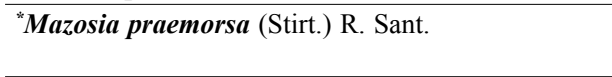 & $6,10,14$ & $\begin{array}{l}6465,6708.2,6719.2,6739,6735,6742,6762, \\
6777,7381.1,7415\end{array}$ \\
\hline "Mazosia pseudobambusae Kalb \& Vězda & 1 & 5918 \\
\hline Mazosia rotula (Mont.) A. Massal. & $1,2,3,4,6,12,13$ & $\begin{array}{l}5866.2,5895,5970,5996,5999,6034.3,6042.1, \\
6173,6182,6203.1,6244.1,6310,6404,6486.3, \\
7076,7312\end{array}$ \\
\hline "Mazosia rubropunctata R. Sant. & $1,3,13,14$ & $\begin{array}{l}5902.3,6192,7110,7146,7248,7339.1,7391, \\
7405.1,7436.1,7436.2,7464\end{array}$ \\
\hline${ }^{*}$ Mazosia tenuissima Lücking \& Matzer & $1,4,6,13,14$ & $\begin{array}{l}5853,5866.1,5898,5906,6270.1,6286.1,6309, \\
6312,6313,6493,7110.1,7166,7194.1,7302, \\
7311,7464.2,7445.1\end{array}$ \\
\hline "Mazosia tumidula (Stirt.) Müll. Arg. & 14 & $7347.1,7391.1,7414,7442$ \\
\hline Microtheliopsis uleana Müll. Arg. & $3,4,6$ & $6178,6254,6304.1,6415$ \\
\hline $\begin{array}{l}\text { "Microtheliopsis uniseptata Herrera-Campos } \\
\text { \& Lücking }\end{array}$ & $1,4,11,14$ & $\begin{array}{l}5862,5881,5907,6259,6292.2,6305.2,6879.1, \\
7382.1\end{array}$ \\
\hline Opegrapha filicina Mont. & $11,13,14$ & $\begin{array}{l}\text { 6791, 7176, 7241, 7268, 7292, 7330, 7330.1, } \\
7385.1,7394,7417\end{array}$ \\
\hline "Opegrapha serusiauxii Lücking & 14 & $7341,7342,7389,7402,7461$ \\
\hline Phyllobathelium firmum (Stirt.) Vězda & 10,13 & $6781.1,7323,7273$ \\
\hline $\begin{array}{l}\text { Phyllobathelium leguminosae (Cavalac. } \\
\text { \& A. A. Silva) Lücking \& Sérus. }\end{array}$ & $\begin{array}{l}1,2,4,7,9,10 \\
12,13\end{array}$ & $\begin{array}{l}5869.2,5998,6065.1,6278,6511.1,6527.1, \\
6658,6663,6684.2,6700.1,6753,7057,7180\end{array}$ \\
\hline Phyllobathelium thaxteri (Vain.) Zahlbr. & 4 & 6318 \\
\hline Phylloblastia bielczykiae Flakus \& Lücking & 12 & 6975.1 \\
\hline "* Phylloblastia excavata P. M. McCarthy & 9,10 & $6678,6684.1,6751$ \\
\hline $\begin{array}{l}\text { *Phyllogyalidea epiphylla (Vězda) Lücking } \\
\text { \& Aptroot }\end{array}$ & $4,7,11$ & $6275.1,6281,6482,6833$ \\
\hline Porina alba (R. Sant.) Lücking & $\begin{array}{l}1,2,4,6,7,10 \\
11,12,13\end{array}$ & $\begin{array}{l}5901,5976,5995.1,5998.1,6040.1,6069,6258, \\
6290,6409,6411,6418,6451,6487.2,6513.4, \\
6532,6542,6544.1,6550,6717.2,6720.1, \\
6722.1,6734.3,6740.2,6755.4,6765,6769.2, \\
6778,6797,6817,6823,6861,6876.1,6883, \\
6914,6948.4,6951,6953,7006,7078.1,7113, \\
7189.1,7223,7327,7239,7295\end{array}$ \\
\hline
\end{tabular}


Table 1. Continued.

\begin{tabular}{|c|c|c|}
\hline Species name & Locality number & Collection number \\
\hline "Porina atriceps (Vain.) Vain. & 10,13 & $6709.1,6740,6765.1,6769,7201.1,7234$ \\
\hline Porina atrocoerulea Müll. Arg. & $1,7,11$ & $5868.1,6439,6478,6550.2,6552,6915$ \\
\hline "Porina barbifera Lücking & 6,9 & $6433,6497,6657,6661.2$ \\
\hline Porina boliviana Flakus \& Lücking & 7 & 6553 \\
\hline Porina conica R. Sant. & 4 & $6241,6284.2$ \\
\hline${ }^{*}$ Porina conspersa Malme & 9 & 6673.2 \\
\hline${ }^{*}$ Porina distans Vězda \& Vivant & 11,12 & $6790,6807,6858.1,6945.3,6999,7075.1$ \\
\hline Porina epiphylla (Fée) Fée & $1,4,11,13$ & $5901.1,5923.1,6280.4,6821,6843,6855,7259$ \\
\hline "Porina fulvella Müll. Arg. & 13 & 7121 \\
\hline "Porina fusca Lücking & 13,14 & $7108,7118,7235,7238,7268,7330.3$ \\
\hline Porina karnatakensis Makhija, Adawadkar \& Patw. & $2,7,10,12$ & $5983,6454,6755.3,6995$ \\
\hline "Porina lucida R. Sant. & $2,4,6,10,12$ & $\begin{array}{l}5952,6252,6280,6411,6709.2,6717,6735.1, \\
6743,6752,7061\end{array}$ \\
\hline Porina nitidula Müll. Arg. & $1,6,9,11,12$ & $5919,5924,6475,6610.1,6635,6834.1,7064$ \\
\hline Porina papillifera (Stirt.) F. Schill. & $1,2,4,6,7,11,12$ & $\begin{array}{l}5896,5933,6031,6249,6265,6414,6439.1, \\
6446.2,6648.1,6482.3,6486.1,6487,6511.2 \\
6871,7070\end{array}$ \\
\hline Porina pseudoapplanata Lücking \& M. Cáceres & $1,7,8,9$ & $5883.1,6441.1,6573,6555,6661.3$ \\
\hline *Porina radiata Kalb, Lücking \& Vězda & $2,4,8,12$ & $\begin{array}{l}5995,6043,6277,6284.3,6304,6317,6564, \\
7070.2\end{array}$ \\
\hline Porina rubentior (Stirt.) Müll. Arg. & $1,2,6,10,11,12$ & $\begin{array}{l}5868,5883,6058.1,6315,6402,6446,6720 \\
6733.1,6740.1,6754.2,6771,6778.1,6810 \\
6840.1,6890,6994\end{array}$ \\
\hline Porina rubescens (Lücking) Hafellner \& Kalb & $4,6,7,11,12,13$ & $\begin{array}{l}6267.1,6433.1,6504,6813,6835,6849,6850, \\
6885,6998,7247\end{array}$ \\
\hline Porina rufula (Kremp.) Vain. & $7,10,12,14$ & $6463,6723.2,6726,7070.3,7401$ \\
\hline Porina subepiphylla Lücking \& Vězda & $2,6,10,11,12$ & $\begin{array}{l}5978,5995.2,6010,6013.1,6077,6410.1,6755, \\
6755.5,6874.1,6936,7001\end{array}$ \\
\hline "Porina subinterstes (Nyl.) Müll. Arg. & 10,12 & 6723,7053 \\
\hline${ }^{* *}$ Porina subnucula Lücking \& Vězda & 2,6 & $5934,5958,6036,6394$ \\
\hline${ }^{* *}$ Porina aff. subpallescens Vězda & 6 & 6430.2 \\
\hline Porina tetracerae (Afzel. ex Ach.) Müll. Arg. & $4,11,12$ & $\begin{array}{l}6284,6858,6873.1,6881,6991,7000,7062.1, \\
7069\end{array}$ \\
\hline Porina tetramera (Malme) R. Sant. & $1,7,11,12$ & $\begin{array}{l}5868.2,5892.1,6552.1,6800,6840,7012 \\
7064.1\end{array}$ \\
\hline${ }^{*}$ Psoroglaena epiphylla Lücking & 11 & 6790.1 \\
\hline $\begin{array}{l}\text { "Sporopodium antonianum Elix, Lumbsch } \\
\text { \& Lücking }\end{array}$ & $2,6,7,8,9$ & $\begin{array}{l}6078.1,6376,6381,6404,6417.1,6442,6450, \\
6462.1,6495.1,6514.3,6579,6634,6666, \\
6698.2\end{array}$ \\
\hline${ }^{*}$ Sporopodium aurantiacum (Müll. Arg.) Lücking & $5,6,14$ & $6332,6381.1,6495.1,7338$ \\
\hline Sporopodium leprieurii Mont. & $\begin{array}{l}1,2,3,4,5,6,7 \\
9,10,12,13,14\end{array}$ & $\begin{array}{l}5925,6076,6171,6273,6344,6365,6400,6417, \\
6459,6664.1,6669,6672.1,6733.2,6992,7087, \\
7299.1,7432\end{array}$ \\
\hline Strigula antillarum (Fée) Müll. Arg. & 2 & 5946.1 \\
\hline${ }^{*}$ Strigula janeirensis (Müll. Arg.) Lücking & $1,2,4,8,12$ & $5863,5957,6253,6594,7077.1$ \\
\hline${ }^{*}$ Strigula macrocarpa Vain. & $1,6,7,8$ & $5859.2,6464,6473,6499,6568$ \\
\hline${ }^{*}$ Strigula maculata (Cooke \& Massee) R. Sant. & $\begin{array}{l}1,2,4,6,7,8,11 \\
12,13\end{array}$ & $\begin{array}{l}5860,5908,5920,5927,5951,5959,5990.1,6028, \\
6044,6071,6246,6283,6283.1,6291,6407.1, \\
6449,6591,6959,7003,7024,7071,7119\end{array}$ \\
\hline
\end{tabular}


Table 1. Continued.

\begin{tabular}{|c|c|c|}
\hline Species name & Locality number & Collection number \\
\hline${ }^{* * *}$ Strigula minuta Lücking & 1 & 5859.1 \\
\hline Strigula nemathora Mont. & $2,8,11,13,14$ & $\begin{array}{l}5929,6012,6052,6558,6590,6603,6853,6884, \\
6959.1,6480,7116,7125,7227,7144.1,7158.1, \\
7187,7201.2,7331,7436\end{array}$ \\
\hline "Strigula nigrocarpa Lücking & 8 & 6583 \\
\hline Strigula nitidula Mont. & 2,8 & 6029,6601 \\
\hline "Strigula obducta (Müll. Arg.) R. C. Harris & $2,4,9,11,12$ & $\begin{array}{l}5966,6257,6279,6610,6641,6650.2,6847, \\
6869.1,6955,6960.1,6993,6995.1,7074, \\
7080.1\end{array}$ \\
\hline${ }^{*}$ Strigula phyllogena (Müll. Arg.) R. C. Harris & $1,2,4,7,9,11,13$ & $\begin{array}{l}5915,6077.3,6283.2,6535,6666.1,6859,7179, \\
7236\end{array}$ \\
\hline${ }^{*}$ Strigula platypoda (Müll. Arg.) R. C. Harris & 4,7 & $\begin{array}{l}\text { 6442.1, 6545, 6474, 6492, 6497, 6513, 6526.1, } \\
6536,6551\end{array}$ \\
\hline "Strigula prasina Müll. Arg. & 2,10 & $6016,6048,6721.1$ \\
\hline Strigula schizospora R. Sant. & $2,11,14$ & $6008,6032.1,6924,7440$ \\
\hline Strigula smaragdula $\mathrm{Fr}$ & $2,4,6,7,8,12$ & $\begin{array}{l}5943,5974,5996.2,6291.1,6422,6460,6546.2, \\
6592,7014,7042\end{array}$ \\
\hline Strigula subtilissima (Fée) Müll. Arg. & 7,10 & $6512.1,6529.1,6776$ \\
\hline "Strigula vulgaris (Müll. Arg.) Lücking & 4,11 & 6247,6864 \\
\hline Tapellaria intermedia Flakus \& Lücking & 1,6 & 5878,6456 \\
\hline Tricharia longispora Kalb \& Vězda & 9 & 6700 \\
\hline Tricharia vainioi $\mathrm{R}$. Sant. & $1,7,9,12,13$ & $5875,5879.2,6493.1,6660,6689,7068,7366$ \\
\hline Trichothelium africanum Lücking & 9 & $6611.1,6637$ \\
\hline Trichothelium alboatrum Vain. & 2 & 5999.3 \\
\hline Trichothelium annulatum (P. Karst.) R. Sant. & $4,6,9,12$ & $6249.1,6263,6433.2,6647.2,7058$ \\
\hline Trichothelium argenteum Lücking \& L. I. Ferraro & $6,7,9$ & $6440,6477,6456,6506,6648,6683,6693$ \\
\hline${ }^{*}$ Trichothelium bipindense F. Schill. & $1,6,9,11,14$ & $\begin{array}{l}5876,5879,5887,6403,6433.4,6461.2,6490, \\
6611,6796,7341.2,7354,7383.1\end{array}$ \\
\hline Trichothelium epiphyllum Müll. Arg. & $1,2,13$ & $5851.1,5854.2,6020.1,6035,7242.1,7263$ \\
\hline 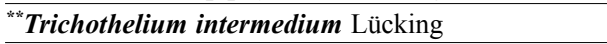 & 13 & 7213 \\
\hline 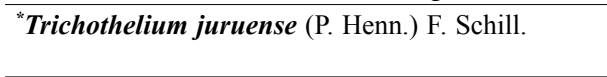 & $1,6,11,13,14$ & $\begin{array}{l}5854.1,5914,6476,6946.2,6962,7148,7198.2, \\
7383\end{array}$ \\
\hline${ }^{*}$ Trichothelium minus Vain. & 12 & $6970,7067.1$ \\
\hline${ }^{*}$ Trichothelium minutum (Lücking) Lücking & 13 & 7174 \\
\hline${ }^{*}$ Trichothelium pallescens (Müll. Arg.) F. Schill. & $1,2,6,7,9,12,13$ & $\begin{array}{l}5879.1,5981,5997,6026,6031.1,6433.3,6439 \\
6453,6490.1,6518,6538,6622,6627,6639 \\
6699.1,6988,7026,7086,7213\end{array}$ \\
\hline "Trichothelium porinoides Vězda & 6 & 6388 \\
\hline Trichothelium sipmanii Lücking & $1,6,11,13$ & $5867.2,6425,6430.1,6461,6835.1,7192$ \\
\hline Trichothelium subargenteum Flakus \& Lücking & $2,4,6,13$ & $6026.1,6264,6433,7208.1$ \\
\hline "Trichothelium ulei (Henn.) Höhn. & 13 & $7129,7169,7198$ \\
\hline
\end{tabular}

P. papillifera, P. rubentior, P. rubescens, $P$. subepiphylla, Sporopodium leprieurii, Strigula obducta, Trichothelium argenteum and T. juraense. Other species found on the study area belong to the lowland to submontane element (29 species, $16 \%$ ), a group possessing a broad altitudinal distribution
(13 species, 7\%) according to Lücking (1997, 2000), or a group of taxa not evaluated previously, with unknown vertical distribution patterns (59 species, 33\%).

Most of the Jaccard's similarity indexes were below 0.25 within group (Table 2), highlighting 


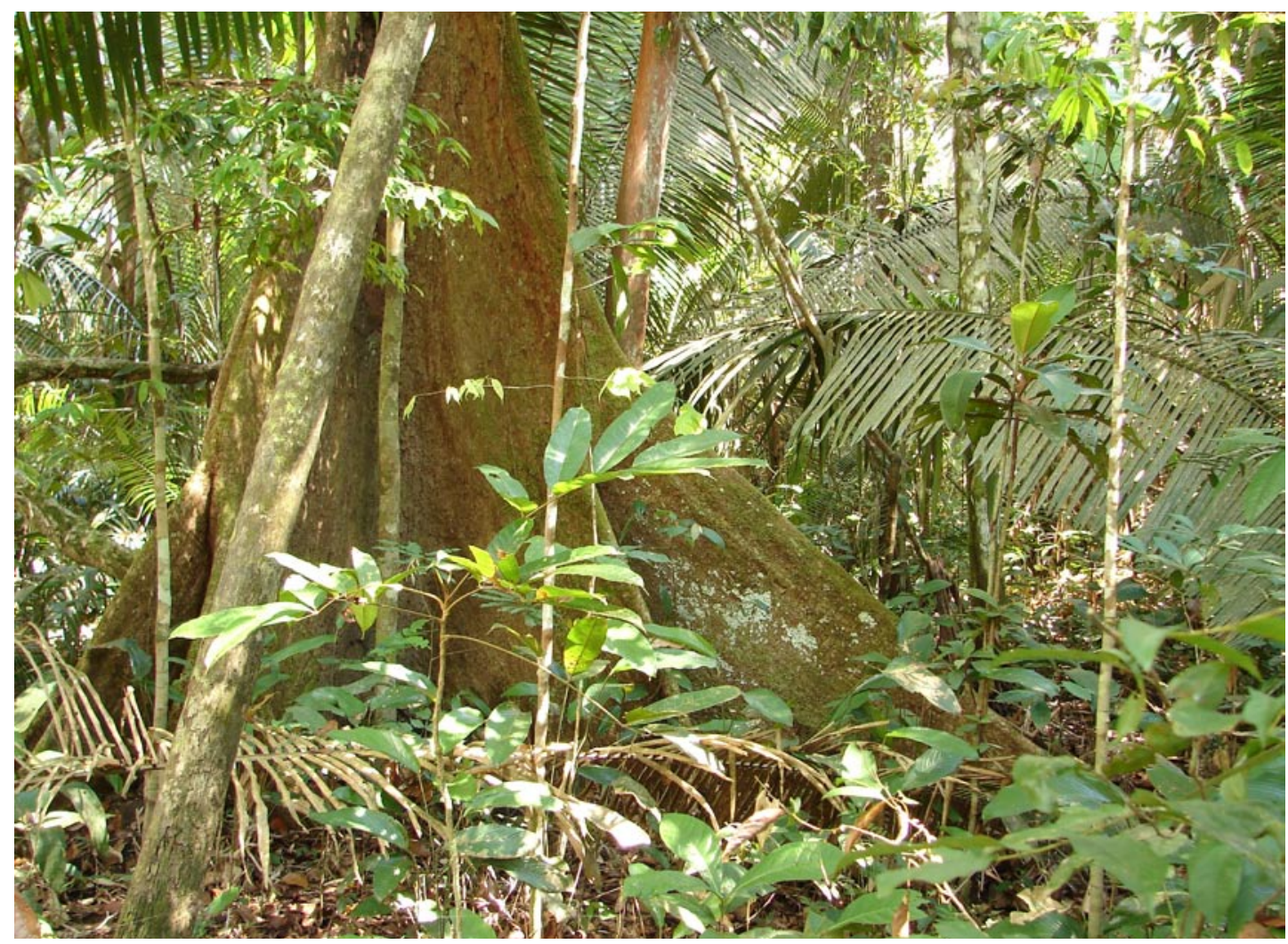

Fig. 3. The site with the highest foliicolous lichen diversity in black-water-flooded Amazon forest (Igapó) of eastern Pando near Santa Cruzito.

the high beta diversity of foliicolous lichens in the study area. Non-metric multidimensional scaling (NMDS) ordination separated the species into three groups (Fig. 4). The resulting pattern was confirmed by hierarchical cluster analysis showing three different clusters (Fig. 5). The first one (A) is formed by site 8 , the second one (B) by sites 1,2 , $4,6,7,9,11$ and 12, and the last one (C) by sites 10,13 and 14. To some extent the three groups are positively correlated with the forest types according to Navarro and Ferreira (2007).

Cluster A is represented only by one site with 20 species (11\%) located in terrestrial Amazon forest of northeast Pando. Its characteristic species composition include lichens rarely noted at the rest of the sites: Calopadia foliicola, $\mathrm{Ba}$ cidina pallidocarnea, Echinoplaca campanulata, Lyromma dolicobelum, Porina pseudoapplanata,
P. radiata, Strigula macrocarpa, S. nigrocarpa and $S$. nitidula.

Clusters B and C form a separate group of riverine forests clearly separated from the first one. Cluster $\mathrm{C}$ combines sites in black-waterflooded Amazon forest (Igapó) of eastern Pando (Precambrian Shield region). Of the 100 species ( $56 \%$ ) found in that forest, many are found exclusively in this vegetation type: Arthonia obrygniae, A. palmulacea, Bacidina hypophylla, Byssoloma amazonicum, B. humboldtianum, Caleniopsis conspersa, C. laevigata, Chroodiscus australiensis, Cryptothecia filicina, Eugeniella leucocheila, E. psychotriae, Fellhanera punctata, F. verrucifera, Mazosia tumidula, Opegrapha serusiauxii, Porina fulvella, P. fusca, Trichothelium minutum and T. ulei. Navarro and Ferreira (2007) classified site 10 as Várzea forest, but the lichen species 
Table 2. Jaccard's similarity index (upper diagonal), number of species shared between study sites (lower diagonal) and number of species per site (diagonal).

\begin{tabular}{|c|c|c|c|c|c|c|c|c|c|c|c|c|c|c|}
\hline $\begin{array}{l}\text { Site } \\
\text { No. }\end{array}$ & 1 & 2 & 3 & 4 & 5 & 6 & 7 & 8 & 9 & 10 & 11 & 12 & 13 & 14 \\
\hline 1 & 51 & 0.317 & 0.167 & 0.261 & 0.089 & 0.310 & 0.333 & 0.164 & 0.189 & 0.159 & 0.297 & 0.301 & 0.295 & 0.149 \\
\hline 2 & 26 & 57 & 0.078 & 0.329 & 0.063 & 0.208 & 0.270 & 0.132 & 0.146 & 0.194 & 0.229 & 0.295 & 0.229 & 0.104 \\
\hline 3 & 9 & 5 & 12 & 0.116 & 0.100 & 0.164 & 0.043 & 0.143 & 0.065 & 0.139 & 0.018 & 0.057 & 0.091 & 0.109 \\
\hline 4 & 18 & 23 & 5 & 36 & 0.070 & 0.218 & 0.259 & 0.077 & 0.159 & 0.161 & 0.246 & 0.356 & 0.200 & 0.090 \\
\hline 5 & 5 & 4 & 2 & 3 & 10 & 0.113 & 0.068 & 0.000 & 0.044 & 0.083 & 0.019 & 0.059 & 0.065 & 0.135 \\
\hline 6 & 26 & 20 & 10 & 17 & 7 & 59 & 0.171 & 0.145 & 0.157 & 0.189 & 0.182 & 0.212 & 0.224 & 0.187 \\
\hline 7 & 22 & 20 & 2 & 15 & 3 & 14 & 37 & 0.140 & 0.254 & 0.158 & 0.206 & 0.266 & 0.172 & 0.075 \\
\hline 8 & 10 & 9 & 4 & 4 & 0 & 10 & 7 & 20 & 0.056 & 0.021 & 0.066 & 0.103 & 0.070 & 0.045 \\
\hline 9 & 14 & 12 & 3 & 10 & 2 & 13 & 15 & 3 & 37 & 0.100 & 0.123 & 0.266 & 0.135 & 0.075 \\
\hline 10 & 11 & 14 & 5 & 9 & 3 & 14 & 9 & 1 & 6 & 29 & 0.104 & 0.217 & 0.188 & 0.182 \\
\hline 11 & 22 & 19 & 1 & 16 & 1 & 16 & 14 & 4 & 9 & 7 & 45 & 0.271 & 0.258 & 0.133 \\
\hline 12 & 22 & 23 & 3 & 21 & 3 & 18 & 17 & 6 & 17 & 13 & 19 & 44 & 0.184 & 0.069 \\
\hline 13 & 28 & 24 & 7 & 18 & 5 & 24 & 16 & 6 & 13 & 16 & 24 & 18 & 72 & 0.315 \\
\hline 14 & 13 & 10 & 6 & 7 & 7 & 17 & 6 & 3 & 6 & 12 & 11 & 6 & 29 & 49 \\
\hline
\end{tabular}

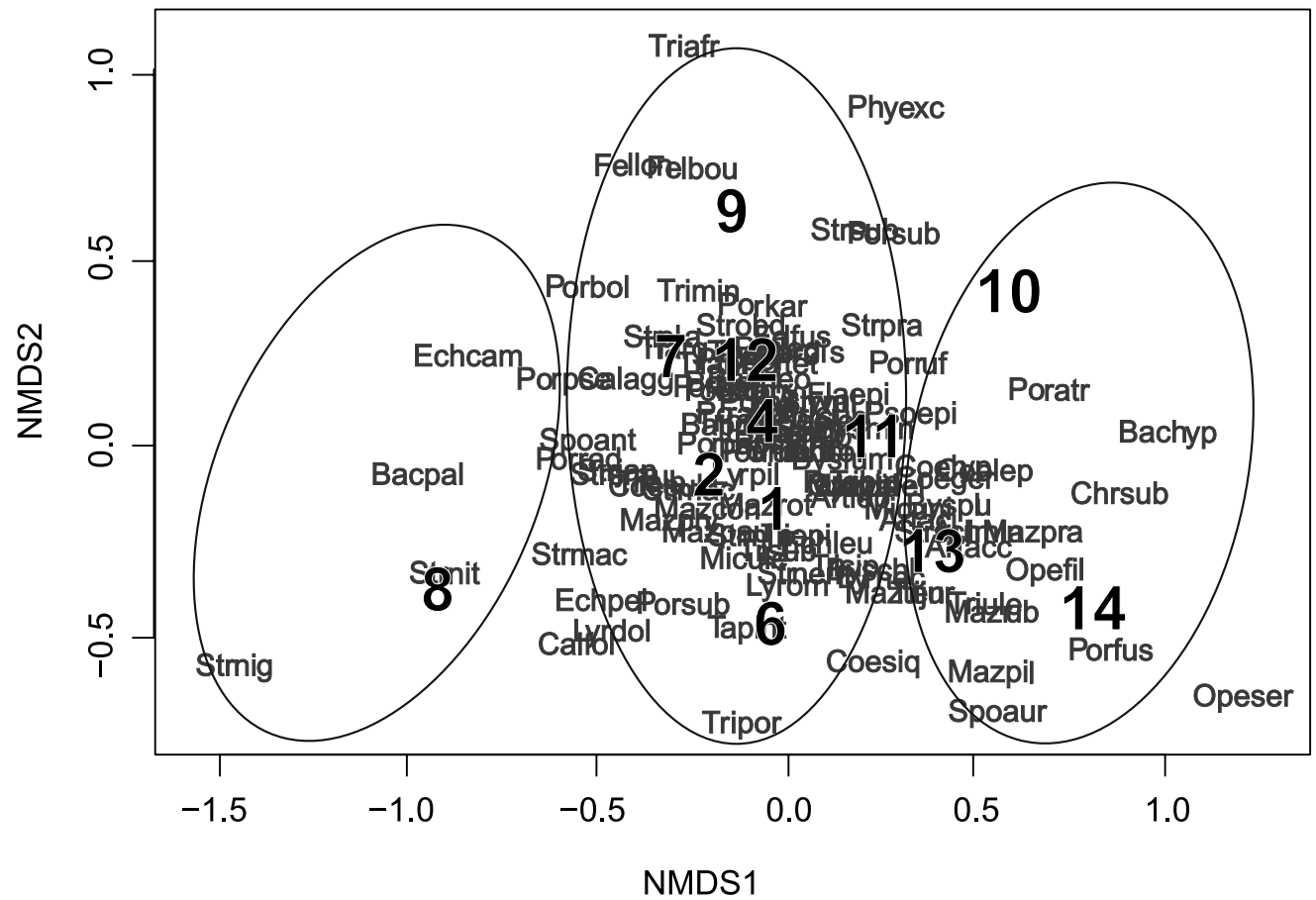

Fig. 4. Non-metric multidimensional scaling (NMDS) of the study sites based on foliicolous lichen composition using Jaccard's dissimilarity index as distance measure. Circle at left surrounds terrestrial forest, in the middle of which is white-water-flooded forest (Várzea); circle at right surrounds black-water-flooded forest (Igapó). Species symbols consist of the first three letters of the generic name and the first three of the epithet. 
0.60

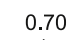

0.80

A

0.70
B

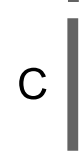

0.90

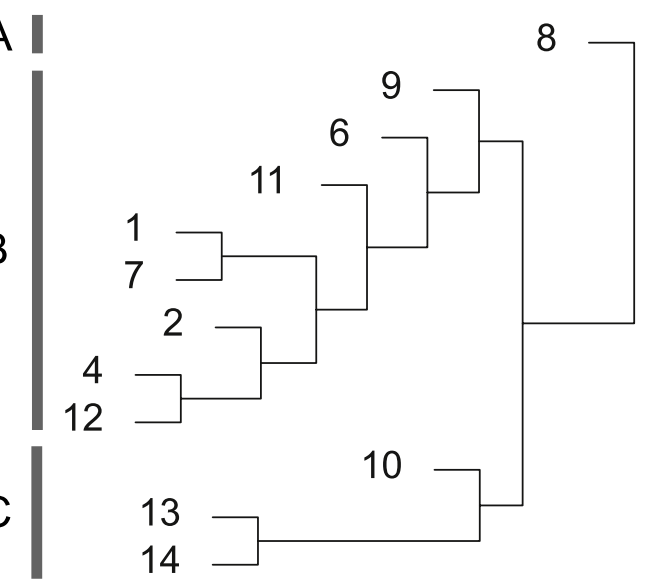

Fig. 5. Hierarchical cluster analysis of the study sites based on foliicolous lichen composition analysis from binary data using average linkage agglomeration. $\mathrm{A}$ - terrestrial forest, $\mathrm{B}$ white-water-flooded forest (Várzea), C - black-water-flooded forest (Igapó).

composition most resembled that of sites 13 and 14 ; this, together with its location near a blackwater river (río Madre de Manupare) suggests that the site is more probably Igapó forest.

Cluster B contains mainly white-water-flooded Amazon forests (Várzea) from western Pando and a few anthropogenically disturbed forests (sites 1, 6, 7). It contains 143 species: $116(64 \%)$ from sites in Várzea forest and 27 (15\%) from anthropogenically disturbed forests. The foliicolous lichen biota of Várzea forest shares many species in common with the other forest types but also contains some exclusive species such as Asterothyrium rondoniense, Aulaxina microphana, Byssolecania variabilis, Caprettia confusa, Fellhanera bouteilei, Gyalectidium laciniatum, Porina distans, P. tetracerae, Strigula obducta and S. vulgaris. The anthropogenically disturbed forests have few exclusive lichens.

The greater diversity of foliicolous lichens in Várzea forest (143 species) as compared with Igapó forest (100 species) might be explained by the higher diversity of vascular plants in the whitewater-flooded forests than in black-water-flooded ones (Navarro \& Maldonado 2002; Navarro 2003;
Mostacedo et al. 2006), which provides more species potentially useful for lichens as a substrate. Fewer species (20) were observed in terrestrial forest (site 8). As commonly known, however, riverine forests (Várzea or Igapó) are much more homogenous floristically than terrestrial high forests (e.g., Mostacedo et al. 2006). The likely explanation for the disparity in numbers is undersampling; only a few sites in terrestrial forest were visited.

It is puzzling that the richest site in the study (with 72 species) is located in Igapó forest, which is considered to be the poorest in vascular plants. A possible explanation is its location in the Precambrian Shield region, the biologically richest and least-disturbed area of forest in the whole Bolivian Amazon, possibly preserving a greater variety of foliicolous lichens.

To evaluate possible undersampling, the Chao 2 estimator (Chao et al. 2009) was calculated for 558 species records from 14 sites. It estimated the potential total number of species in the studied area at $c a 214$ species. Thus we may have collected $84 \%$ of the species expected to be found in the area. The number of analyzed sites was not uniform across all types of forest, however, so these results should be taken as preliminary. To propose a reliable set of distribution patterns for the foliicolous lichens in the forest vegetation of Pando, more sites need to be investigated, especially those in terrestrial forests and in the Precambrian Shield region.

ACKNOWLEDGEMENTS. I am very grateful to Silvia C. Gallegos (La Paz) and Pamela Rodriguez Flakus (Frankfurt) for their kind help with the statistical analyses and for valuable comments, Dr. hab. Martin Kukwa (Gdańsk) for reviewing the manuscript and for constructive comments, and to Rosa I. Meneses Q. (La Paz), Director of Herbario Nacional de Bolivia, Instituto de Ecología, Universidad Mayor de San Andrés, and Vincente Chura Palli (Cobija), Director of Reserva Nacional de Vida Silvestre Amazónica for their generous cooperation. I thank Dr. Harrie J. M. Sipman (Berlin) for his kind hospitality during my visit to herbarium $\mathrm{B}$, and Celso Bismarck Ruiz (Chive) and others for their help during fieldwork. This research received support from the Polish National Science Center (NCN) for 2008-2011 (grant no. N N303 345335), the SYNTHESYS Project (DE-TAF-4939) financed by the European Commission's Sixth Frame- 
work Programme, and from the W. Szafer Institute of Botany of the Polish Academy of Sciences through its statutory fund.

\section{REFERENCES}

ANONYMOUS 1997. ZONISIG. Zonificación agroecológica y socioeconómica y perfil ambiental del departamento de Pando. Ministerio de Desarrollo Sostenible y Medio Ambiente, La Paz, Bolivia.

Aptroot A., Diederich P., SÉRUSiaux E. \& SipMAN H. J. M. 1997. Lichens and Lichenicolous Fungi from New Guinea. Biblioth. Lichenol. 64: 1-220.

Boonpragob K., Homchantara N., Coppins B. J., MCCARTHY P. M. \& WOLSELEY P. A. 1998. An introduction to the lichen flora of Khao Yai National Park, Thailand. Botanical Journal of Scotland 50(2): 209-219.

BReuss O. \& NeuWIRTH G. 2007. Flechtenfunde im Bosque Esquinas, Costa Rica. Linzer Biol. Beitr. 39(1): 557-569.

Chao A., Colwell R. K., Lin C.-W. \& Gotelli N. J. 2009. Sufficient sampling for asymptotic minimum species richness estimators. Ecology 90(4): 1125-1133.

CRAWLEY M. J. 2013. The R book. Second edition. John Wiley \& Sons, Ltd., Chichester, UK.

Davidson E. A., de Araújo A. C., Artaxo P., Balch J. K., Brown I. F., Bustamante M. M. C., Coe M. T., DeFries R. S., Keller M., Longo M., Munger J. W., Schroeder W., Soares-Filho B. S., Souza C. M. \& WofsY S. C. 2012. The Amazon basin in transition. Nature 481: 321-328.

FARKAS E. 2010. Notes and schedae to Lichenes Delicati Exsiccati Editae in memoriam Antonín Vězda (1920-2008), Fasc. 1. Acta Bot. Hung. 52(3-4): 331-340.

FERraro L. I. 2002. Contribution to the knowledge of foliicolous lichens of Bolivia. Mitt. Inst. Allg. Bot. Hamburg 30-32: 47-48.

FERrARo L. I., LÜCKING. R. \& SÉRUSIAUX E. 2001. A world monograph of the lichen genus Gyalectidium (Gomphillaceae). Bot. J. Linn. Soc. 137: 311-345.

FLAKUS A. \& FARKAS E. 2013. A contribution to the taxonomy of Lyromma (Lyrommataceae, lichenized Ascomycota) with a species key. Mycotaxon 124: 127-141.

FLAKUS A. \& LÜCKING R. 2008. New species and additional records of foliicolous lichenized fungi from Bolivia. $\mathrm{Li}$ chenologist 40(5): 423-436.

Herrera-CAMPos M. A. \& LÜCKING R. 2002. The foliicolous lichen flora of Mexico. I. New species from Los Tuxtlas Tropical Biology Station, Veracruz. Lichenologist 34(3): 211-222.

Herrera-Campos M. A., Colín P. M., BÁrcenas Peña A. \& LÜCKING R. 2004. The foliicolous lichen flora of Mex- ico. III. New species from Volcán San Martín Tuxtla (Sierra de Los Tuxtlas), Veracruz, with notes on Fellhanera santessonii. Phyton (Horn) 44: 167-185.

IBISCH P. L. \& MÉRIDA G. (eds.). 2004. Biodiversity: the richness of Bolivia. State of knowledge and conservation. Ministry of Sustainable Development. Editorial FAN, Santa Cruz de la Sierra, Bolivia.

JACCARD P. 1908. Nouvelles recherches sur la distribution florale. Bull. Soc. Vaud. Sci. Nat. 44: 223-270.

Josse C., Navarro G., Comer P., Evans R., FABER-LANGENdoen D., Fellows M., Kittel G., Menard S., Pyne M., Reid M., Schulz K., Snow K. \& Teague J. 2003. Ecological Systems of Latin America and the Caribbean: A Working Classification of Terrestrial Systems. NatureServe, Arlington.

LÜCKING R.1995. Biodiversity and conservation of foliicolous lichens in Costa Rica. In: C. SCHEIDEGGER, P. A. WOLSELEY \& G. THOR (eds), Conservation Biology of Lichenised Fungi, pp. 63-92. Mitteilungen der Eidgenössischen Forschungsanstalt für Wald, Schnee und Landschaft, Birmensdorf, Switzerland.

LÜCKING R. 1997. The use of foliicolous lichens as bioindicators in the tropics, with special reference to the microclimate. Abstr. Bot. (Budapest) 21(1): 99-116.

LÜCKING R. 1998. Foliicolous lichens and their lichenicolous fungi collected during the Smithsonian International Cryptogamic Expedition to Guyana 1996. Trop. Bryol. 15: $45-76$.

LÜCKING R. 2000. List of environmental index values for foliicolous lichens. [Version: February 29th, 2000]. http:// www.bio.uni-bayreuth.de/planta2/ass/robert/lichens/listindi.html

LÜCKING R. 2008. Foliicolous lichenized fungi. Fl. Neotrop. Monogr. 103: 1-867.

LÜCKING R. \& KALB K. 2000. Foliikole Flechten aus Brasilien (vornehmlich Amazonien), inklusive einer Checkliste und Bemerkungen zu Coenogonium und Dimerella (Gyalectaceae). Bot. Jahrb. Syst. 122: 1-61.

LÜCKING R. \& VĚZDA A. 1998: Taxonomic studies in foliicolous species of the genus Porina (lichenized Ascomycotina: Trichotheliaceae) - II. The Porina epiphylla group. Willdenowia 28: 181-225.

MCCARTHY P. M. 2010. New and interesting foliicolous lichens from Australia. Australas. Lichenol. 66: 4-15.

MINCHIN P. R. 1987. An evaluation of relative robustness of techniques for ecological ordinations. Vegetatio 69: 89-107.

Montambault J. R. (ed.) 2002. Informes de las evaluaciones biológicas Pampas del Heath, Perú, Alto Madidi, Bolivia y Pando, Bolivia. RAP Bulletin of Biological Assessment 24: 1-124.

Mostacedo B., Balcazar J. \& Montero J. C. 2006. Tipos de 
bosque, diversidad y composición florística en la Amazonia sudoeste de Bolivia. Ecol. Bolivia 41(2): 99-116.

NAVARro G. 2003. Tipología fluvial y vegetación riparia amazónica en el departamento de Pando (Bolivia). Revista Boliviana de Ecología y Conservación Ambiental 13: 3-29.

NAVArro G. 2011. Clasificación de la Vegetación de Bolivia. Centro de Ecología Difusíon Simón I. Patiño, Santa Cruz, Bolivia.

NAVARRo G. \& FERREIRA W. 2007. Mapa de Vegetación de Bolivia a escala 1:250.000. The Nature Conservancy (TNC). Ed. digital. Santa Cruz de la Sierra.

NAVARRo G. \& MALdonado M. 2002. Geografía ecológica de Bolivia: vegetación y ambientes acuáticos. Centro de Ecología Difusíon Simón I. Patiño, Santa Cruz, Bolivia.

Orange A., James P. W. \& White F. J. 2001. Microchemical methods for the identification of lichens. British Lichen Society, London.

PAPONG K., BoONPRAGOB K. \& LÜCKING R. 2007. New spe- cies and new records of foliicolous lichens from Thailand. Lichenologist 39(1): 47-56.

Rivas Plata E. \& LÜCKING R. 2013. High diversity of Graphidaceae (lichenized Ascomycota: Ostropales) in Amazonian Perú. Fung. Diversity 58(1): 13-32.

Rivas Plata E., LÜCKing R., Aptroot A., Sipman H. J. M., Chaves J. L., Umaña L. \& Lizano D. 2006. A first assessment of the Ticolichen biodiversity inventory in Costa Rica: the genus Coenogonium (Ostropales: Coenogoniaceae), with a world-wide key and checklist and a phenotype-based cladistic analysis. Fung. Diversity 23: 255-321.

SANTESSON R. 1952. Foliicolous Lichens I. A revision of the taxonomy of the obligately foliicolous, lichenized fungi. Symb. Bot. Upsal. 12(1): 1-590.

Sipman H. J. M. 1993. Lichens from Mount Kinabalu. Trop. Bryol. 8: 281-314.

VĚZDA A. 1975. Foliicole Flechten aus Tanzania (Ost-Afrika). Folia Geobot. Phytotax. 10: 383-432.

Received 24 June 2013 